\title{
Poésie et Autobiographie Ressemblances et différences
}

\author{
Recherche présentée par \\ Tamer SAYED ABD EL-TAWAB
}




\section{1- Problème de définition}

Presque tous les dictionnaires définissent l'autobiographie par "Biographie de l'auteur écrit par lui-même". Or, le mot "biographie" ne donne pas de vraie définition sur la forme ou le genre de l'autobiographie. Reste encore cette question de genre qui donne une certaine ambigüité à l'autobiographie.

Le Dictionnaire Larousse 2010 définit l'autobiographie comme "Biographie d'une personne écrite par elle-même"1. Une définition qui semble la même dans Le Petit Robert de la langue française 2009 qui la définit comme "Biographie de l'auteur faite par lui-même". ${ }^{2}$

Etymologiquement parlant, le mot autobiographie est formé de trois racines grecques : Autos qui signifie soi-même, Bio dont le synonyme est la vie et enfin Graphein qui veut dire écrire. D'après cette étymologie, on peut dire que l'autobiographie signifie l'écriture de soi-même ou le fait que l'écrivain écrit luimême sa biographie. L'étymologie garde encore la forme et le genre, d'après lesquels une autobiographie doit être écrite, comme vagues et non-définis.

\footnotetext{
1 - Larousse 2009, Paris, P.80

2 - Le Petit Robert de la langue française, Millésime, 2009 Paris, P. 182
} 
Quant aux encyclopédies, la problématique du genre de l'autobiographie reste encore évidente . Dans "Encyclopédia Universalis" l'auteur met l'accent sur le problème d'une définition du genre de l'autobiographie : "Peut-on définir l'autobiographe? Aucun critère purement linguistique ne semble pertinent. Rien ne distingue à priori autobiographie et roman à la première personne". ${ }^{3}$ Cependant l'essai met en cause la définition de Philippe Lejeune dans son livre le pacte autobiographique qui définit l'autobiographie comme "un récit rétrospectif en prose qu'une personne réelle écrit sur sa propre vie" :

Les écrits autobiographiques de Simone de Beauvoir ne sont pas exclusivement le récit d'une vie individuelle, les Mémoires d'outre-tombe ne sont pas rétrospectifs, les écrits autobiographiques de Leiris ou d'Adamov ne sont pas des récits, le montage de son journal dans Le Temps immobile n'est pas un récit mais il est rétrospectif. Une vie ordinaire de Georges Perros est une autobiographie en vers. Les Mots de Sartre ne sont pas le récit d'une expérience, etc. ${ }^{4}$

L'auteur de cet essai renvoie, donc, le lecteur, à la hâte, à la possibilité d'une poésie autobiographique ou une autobiographie en vers. Sans entrer dans le vif du sujet, l'auteur met sous les

\footnotetext{
3 - Encyclopidia Universalis, S.A 2002, paris, P. 472

4 - Encyclopidia Universalis, S.A 2002, paris, P. 472
} 
yeux de son lecteur l'exemple de Une vie ordinaire de Georges Perros.

Pour les théoriciens, la problématique de la définition de l'autobiographie n'est pas différente des dictionnaires ou des encyclopédies. Dans son livre Le pacte autobiographique Philippe Lejeune essaie de donner une définition probable de l'autobiographie tout en reconnaissant l'existence du problème de définition :

"Légèrement modifié, la définition de l'autobiographie serait : Récit rétrospectif en prose qu'une personne réelle fait de sa propre existence, lorsqu'elle met l'accent sur sa vie individuelle, en particulier l'histoire de sa personnalité" 5

Cette définition met devant nous une série de problématiques à discuter concernant la forme du langage utilisé dans l'écriture de l'autobiographie, le genre littéraire adopté pour une telle écriture...etc. D'après cette définition lejeunienne, l'autobiographie doit, en premier lieu, adopter un système narratif où l'autobiographe suit un certain ordre chronologique. L'utilisation du temps est alors calculée et n'est pas gratuite. En deuxième lieu, l'autobiographie doit être écrite en prose pour que l'auteur ne soit pas soucieux des rimes ou d'autres règles de la poésie et arrive tout facilement à se remémorer des moments passés. En troisième lieu, l'autobiographie est un récit écrit par

\footnotetext{
${ }^{5}$-Le Pacte autobiographique, Philippe Lejeune, Paris, seuil 1975, p.14
} 
une personne réelle, ceci nécessite que le "je" auteur soit lié au "je" narrateur par un pacte autobiographique dans lequel l'auteur s'engage implicitement à dire la vérité dès le début de son œuvre. En quatrième lieu, l'autobiographie a sa forme propre à elle qui diffère des autres écrits personnels comme le journal intime ou les mémoires.

D'une autre part, la poésie partage cette difficulté de définition, puisque presque tous dictionnaires ne font pas la distance entre la poésie en vers et la poésie en prose. A titre d'exemple, le dictionnaire Hachette définit la poésie comme "forme d'expression caractérisée par une utilisation harmonieuse des sons et des rythmes du langage (notamment dans le vers) et par une grande richesse d'image" ${ }^{\prime 6}$. De même l'encyclopédie universalis évoque le même problème de définition comme elle a déjà fait avec l'autobiographie. Nous trouvons l'article parlant du mot "poésie" commence par : "La poésie est d'autant plus difficile à définir qu'elle recouvre une pratique très diversifiée, plus qu'un genre particulier. ${ }^{17}$

\section{$\underline{\text { L'écriture autobiographique (prose et vers) et les autres }}$}

\section{formes de l'écriture de soi}

\section{$\underline{\text { Le journal intime }}$}

\footnotetext{
6 - Hachette 2001,

7 - Encylcopédia Universalis, corpus 18, Paris 2002, P.401
} 
Les écritures autobiographiques sont diverses voire divergentes, nous essayerons, dans ce qui suit, de proposer une vue d'ensemble sur la différence entre ces écritures prosaïques et poétiques. D'abord, nous avons le journal intime. En fait, le journal intime se définit comme l'écriture quotidienne des événements personnels. Autrement dit, c'est un texte rédigé de façon régulière ou intermittente racontant les actions datées à une certaine période de la vie.

Dans son livre Les journaux intimes, ${ }^{8}$ Michèle Leleu propose pour la première fois le terme "diariste" pour signifier l'auteur du journal intimes, qui est en fait, emprunté à l'anglais "diarist" et reformé sur le vieux mot français "diaire". De son côté, Béatrice Didier signale dans son livre, Le journal intime ${ }^{9}$, quelques éléments faisant la différence entre l'autobiographie et le journal intime. D'abord, le journal intime se caractérise par sa périodicité. Cela veut dire qu'un texte de journal intime est écrit souvent au jour le jour. Leleu note, cependant, qu'il y a des diaristes qui supposent un certain recul par rapport à l'action et puis, ils commencent toute suite la rédaction après une courte période, et il y a aussi les "raffinés" qui attendent la situation idéale pour écrire. Cela montre que l'écriture du journal intime suppose une simultanéité ou un petit retard par rapport à

\footnotetext{
8 - Les journaux intimes, Michèle LELEU, Paris, PUF 1952

9 - Le journal intime, Béatrice DIDER, Paris, PUF 2002
} 
l'action. Cette périodicité d'écriture réduit la distance entre le vécu et le narré à l'apposé de l'autobiographie qui devrait résumer le chemin de vie dans quelques pages. Cette périodicité met encore en évidence une autre différence entre le journal intime et l'autobiographie. En fait, l'autobiographie devrait subir du temps en temps des failles de mémoire, ce qui est peu survenu avec l'écriture du journal intime car le diariste relate au fur et à mesure ce qui lui arrive. En deuxième lieu, le journal intime se caractérise par le goût du secret. Cette caractéristique contrarie l'une des visées essentielles de l'autobiographie où l'autobiographe s'adresse à un public pour lui transmettre un message à travers le récit de sa vie : prouver une idée quelconque, se défendre ou même s'attaquer comme dans le cas des rêveries du promeneur solitaire, de Rousseau,...etc. En troisième lieu la forme du journal intime n'est pas figée, c'est-àdire que nous pouvons voir un journal intime sous forme de paragraphe, de croquis, des passages en prose ou en vers...etc. Cette dernière forme nous mène à la relation entre le journal intime et la poésie, ce que nous allons éclaircir dans le point suivant.

\section{Le journal intime et le journal poétique}

En général le journal intime et la poésie ont en commun la représentation de la vie personnelle. Mais à partir de XIXème 
siècle, les critiques assistent à un nouveau type de journal intime, c'est ce qu'on appelle le journal poétique. L'évolution de ce type de journal commence dès la fin du XVIIIème siècle avec le journal de Lucile Desmoulins (1788-1793), puis au XIXème siècle avec le journal d'Amiel (1839-1881) où l'on trouve des passages lyriques et méditatifs.

Quelques fois les diaristes intègrent des passages en vers parmi les passages en prose. En fait, selon Michel Braud "le journal intime se présente comme l'aveu direct d'une expérience personnelle, les vers retranscrits n'en sont l'écho que par emprunt, c'est-à-dire par la ré-énonciation d'un discours préexistant". ${ }^{10}$ Selon lui, le statut du journal poétique devient plus clair lorsque le diariste essaie de poétiser l' expérience réelle qu'il a vécue pour l'écrire en vers. C'est le cas, par exemple, de Claude Roy qui écrit dans son journal intime un poème qui prend la date du 21 septembre 1992 :

Un milieu de matinée à la fin de septembre Le soleil s'est levé loin très tard très lentement tout de l'autre côté de l'épaisseur de brume Une clarté de perle fondue dans l'air humide ${ }^{11}$

\footnotetext{
${ }^{10}$ - Michel Braud, les poèmes des jours, Journal personnel et journal poétique, in L'irressemblance poésie et autobiographie, Modernités 24, presse universitaire de Bordeaux, 2007. P.78

11 - Claude Roy, les rencontres des jours, Gallimard, "folio» 1996, in "I'irressemblance poésie et autobiographie, ibid, $\mathrm{p} 78$
} 
Ou bien dans comme dans le journal intime d'Henri-Frédéric Amiel où nous lisons :

(7heures matin.) Fort mal dormi, dans ma chambrette étranglée et dans mon lit mal fait

Dans mon petit lit dur,

Étouffé dans la boîte

De ma chambrette étroite,

Pendue à son vieux mur

Je me sens l'âme lourde ${ }^{12}$

Quelques diaristes respectent de même la chronologie de leurs expériences quotidiennes. Le journal poétique par exemple qui s'intitule Le pain quotidien de William Cliff, publié en 2006, suit une longue chronologie, en vers, montrant des expériences réelles que le poète a vécues. C'est aussi le cas de Jean Cayrol dans son recueil Poésie-Journal publié en 1977 où l'on observe dès le titre cette insistance de la part du diariste poète d'établir une ressemblance entre la poésie et le journal intime. Le journal poétique se voit encore dans ce qu'on appelle récemment "les journaux-feuilletons" à publication intercalée. C'est comme l'œuvre de Philippe Jaccottet dont il a publié en 1985 le premier tome des "Semaisons " couvrant les années 1954-1979 et puis le second tome en 1996 pour les années 1980-1994

\footnotetext{
12 - Journal intime (1839-1881), Amiel, Henri Frédéric, in la ressemblance poésie et autobiographie, Op.Cit, p 79
} 
Le rôle de la prose poétique est évident dans le journal poétique, c'est le cas par exemple de Le cahier vert de Maurice Guérin qui est un journal intime comprenant des paysages et des impressions. L'auteur y mêle des copies des lettres, des notes de lecture, des croquis littéraires et des poèmes en prose. Ou bien c'est comme dans l'exemple de l'œuvre de Yves Charnet intitulé Proses du fils. En fait, cette prose poétique se démarque des autres formes d'écriture par plusieurs marques; à savoir : l'absence de ponctuation, l'usage des blancs et parfois des phrases nominales.

En fin, la ressemblance entre le journal poétique et le journal intime va jusqu'à ce que les diaristes poètes datent leurs poèmes et écrivent des poèmes datés racontant leur journée de jour en jour. La liste est longue de ceux qui ont adopté une telle méthode, nous citons à titre d'exemple : Carnets de Paul de Roux, Une certaine latitude de Lionel Bourg et L'Estran de Denise Le Dantec. ${ }^{13}$

\section{Les mémoires et l'autobiographie}

A la différence de l'autobiographie, le mémorialiste met l'accent sur l'historique d'une vie professionnelle en parallèle avec l'histoire de son pays. La particularité est alors accordée aux

\footnotetext{
13 - Les poèmes des jours, journal personnel et journal intime in la ressemblance poésie et autobiographie, Op.Cit, P (77 à 86)
} 
éléments historiques, puis vient la vie intime de l'auteur en deuxième lieu. En fait, on connaît les mémoires dès l'Antiquité, par exemple, on connaît les mémoires du roi Pyrhus (318-272 avant J.C) où il a mis sa vision de l'art de la guerre. Or, ce genre comme tous les genres littéraires, a évolué au cours des siècles, surtout au XXème siècle qui a vu deux événements historiques majeures, ce sont les deux guerres mondiales. En effet, la seconde guerre mondiale a été une raison forte pour une évolution totale de ce genre. Nous voyons, par exemple, l'apparition de ce qu'on appelle "Carnets de guerre" qui sont des œuvres ayant mélangé le récit personnelle de l'auteur et son expérience vécue pendant la guerre.

\section{Poésie et Histoire}

L'une des questions qui a certainement rapport avec la véracité de la poésie, c'est sa capacité de relater les faits historiques. On verra ici quelques exemples pouvant expliquer ce rapport complexe entre la poésie et l'Histoire.

\section{A- L'épopée}

Dans son livre L'épopée, Daniel Madelénat fait la distinction entre l'écriture de l'Histoire dans un livre d'Histoire et celle dans un poème épique : 
Dans un premier sens plus restreint, le poème épique proprement dit, soumis à ses règles, avec son merveilleux, ses épisodes, etc. C'est l'imitation, en récit, d'une action intéressante et mémorable ; ainsi l'épopée diffère de l'Histoire qui raconte sans limiter; du poème dramatique, qui peint en action ; du poème didactique, qui est un tissu de préceptes ; et des fastes en vers qui ne sont qu'une suite d'événement sans unité ${ }^{14}$.

La première caractéristique est, alors, l'imitation des exploits guerriers ou aventuriers d'une personne ou d'un peuple et ne pas raconter l'histoire exacte. Autrement dit, le poète d'un poème épique n'a pas la même objectivité que l'historien doit suivre en sa rédaction de l'Histoire. Il essaie de sélectionner de l'Histoire ce qu'il convient à l'objectif de son épopée.

Parmi les caractéristiques de l'épopée, c'est le recours à 1'oralité. Le poète devait, à l'époque, réciter des dizaines de vers rythmés et rimés. Il chantait, riait, pleurait et imitait la scène historique qui est le sujet de son poème. L'exemple le plus net, c'est l'épopée homérique.

L'action est aussi l'un des points forts de l'épopée. Normalement, une épopée relate l'histoire d'une action qui rend la gloire à la nation : une action ayant pour objectif d' achever l'unité du peuple, de sauver la patrie des attaques étrangères, de réserver le patriotisme du pays...etc. Donc, l'action centrale est toujours celle qu'on vient d'évoquer, mais il y a aussi d'autres

\footnotetext{
14 - L'épopée, Daniel Madélenat, presse universitaire de France, 1986, Paris. P. 19
} 
actions secondaire qui viennent s'ajouter à l'histoire pour étoffer l'intrigue comme : l'amour, l'aventure, le voyage...etc.

Le personnage est la base de l'épopée, cela veut dire que tout récit narratif épique doit glorifier un tel ou tel personnage. Le héros peut être une personnalité connue dans l'histoire réelle comme dans la chanson de Roland ou un héros fictif qui remonte à une mythe comme les épopées grecques.

\section{B- Témoignage et poésie}

Dans son livre Poétique Aristote fait la distinction entre la poésie et l'Histoire : "La poésie dit le général, l'Histoire le particulier". En fait, beaucoup de théoriciens et de critiques ont soulevé la problématique du témoignage littéraire tout en mettant l'accent sur les textes en prose, oubliant ainsi que la poésie peut aussi de son tour témoigner les événements historiques que le poète vit. Nous allons essayer de donner quelques exemples du témoignage poétique et prouver que c'est à chaque poète de raconter l'événement de sa manière :

- Dans son poème "Poème sur le désastre de Lisbonne", Voltaire commence par éprouver le malheur et la misère du tremblement de terre en général.

O malheureux mortels ! ô terre déplorable !

$\mathrm{O}$ de tous les mortels assemblage effroyable ! 


\begin{abstract}
D'inutiles douleurs éternel entretien !
Philosophes trompés qui criez : «Tout est bien ${ }^{15}$
\end{abstract}

Puis, il précise de quel malheur il parle, et évoque ainsi celle de Lisbonne tout en critiquant la société française qui a l'aire non intéressé et non ému de ce qui s'est passé à Lisbonne :

Lisbonne, qui n'est plus, eut-elle plus de vices

Que Londres, que Paris, plongés dans les délices:

Lisbonne est abîmée, et l'on danse a Paris.

Tranquilles spectateurs, intrépides esprits, ${ }^{16}$

Là, on trouve que le poète témoigne de ce qui s'est passé à Lisbonne et témoigne aussi de l'indifférence de la société française face à ce problème. Or, ce témoignage est un témoignage flou qui manque de date ou des noms des personnalités et qui est basé seulement sur les expériences sociales que le poète a accumulées et son sentiment face au désastre de Lisbonne. Cela veut dire que le poème manque les informations pour qu'il puisse être un témoignage crédible.

\title{
Conclusion
}

Dans cette recherche, on a exposé des certains points communs entre la poésie et les autres sortes de l'écriture de soi et surtout l'autobiographie. On a commencé par la difficulté de la définition qui regroupe les deux. Puis, on a essayé de faire une sorte de lien entre les autres écritures de soi et la poésie. On a vu comment la poésie pourrait aller à l'instar du journal intime

\footnotetext{
15 - Poème sur le déastre de Lisbonne, Voltaire

16 - Ibid
} 
jusqu'à ce qu'on voie ce qu' on appelle le journal poétique. La poésie est, aussi, en lien commun avec les mémoires et on justifié comment des poèmes pourraient retracer la carrière d'une personnalité célèbre ou d'un certain poste social. En plus, vu que le corpus contient une œuvre de deuil, on a vu comment la poésie se rattache à un tel type d'écriture en faisant la comparaison entre elle et les autres types de l'écriture de deuil.

Pour prouver cette capacité de la poésie à retracer les autres types de l'écriture de soi, il fallait montrer sa capacité à raconter le réel, puis que c'est la caractéristique primordiale de toute écriture de soi. On a montré la capacité de la poésie à dire la vérité et on a vu qu'elle exagère souvent même quand elle retrace des événements réels. On a, aussi, donné l'exemple de quelques recueils célèbre retraçant la gloire des autres. 


\section{Bibliographie}

\section{Dictionnaires et Encyclopédies}

Encyclopédie "Universalis", S.A, Paris 2002

\section{Livres critiques}

BAFARO Gorges, L'épopée, éllipses, Paris 1997

CLERC Thomas, Les écrits personnels, Hachette Education, Paris 2001

DUFIEF Pierre-Jean, Les écritures de l'intime, Bréal, SeineSaint-Denis 2001

LEJEUNE Philippe, Le Pacte autobiographique, Seuil, Paris 1975

LEJEUNE Philippe, L'autobiographie en France, Armand Colin, Paris 1971

LEJEUNE Philippe, Signes de vie, Le pacte autobiographique 2, Seuil, Paris 2005

SIMONET-TENANT Françoise, Le journal intime : genre littéraire et écriture ordinaire, Nathan, Paris 2001

\section{Euvres Littéraires}

ERNAUX Annie, La place, Gallimard 1986

HUGO Victor, Euvres complètes (Les châtiments, les contemplations, la légende des siècles, la chanson des rues et des bois, la voix de Guernesey, R. Laffont, Paris 2002

QENEAU Raymond, Chêne et chien, Gallimard, Paris 1969 Revues 
BRAUD Michel et HUGOTTE Valéry, L'irressemblance Poésie et autobiographie, Modernités 24, presse universitaire de Bordeaux 2007

\section{Thèses}

RZGAIEG Najiba, De l'autobiographie à la fiction ou le je(u) de l'écriture, Thèse de doctorat de littérature française, université "Paris-Nord", faculté des lettres, 1995 


\section{الثعر والسيرة الذاتية، تثابه واختلاف}

يتضمن موضوع بحثنا محاولة لإكتثاف العلاقة بين الثعر والسيرة

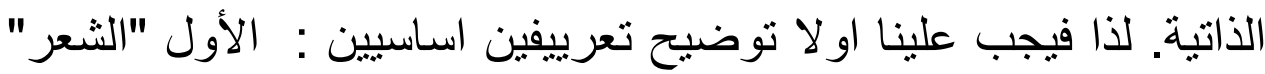
حيث نتعرض في هذا البحث إلى نماذج غير تقليدية للشعر ونحاول تعريف الحدود بين الثعر والنثر. أما التعريف الثاني فهو السيرة الذاتية. نلقي الضوء ايضا في بحثنا على التشابه بين الثعر والأنواع المختلفة للكتابات الذاتية، فمثلاً سوف ندرس التشابه بين الدذكرات اليومية النثرية و المذكرات اليومية الثعرية تلك المذكرات التي تكتب على هيئة قصائد وبعضها يكون مؤرخ بل ان بعض الثعر اء قد دونوا مذاكر اتهم اليومية مؤرخة يوم بيوم على هيئة شعر.

\section{Poetry and autobiography, similarities and differences}

Our research topic includes an attempt to discover the relationship between poetry and autobiography. Therefore, we must first clarify two basic definitions: the first discuss the "poetry". We try to define the boundaries between poetry and prose. The second definition discuss the autobiography. For example, we will examine the similarities between the prose diary and the poetic diary. These notes are wrote in the form of poems, some of which are dated. Some poets have even wrote their daily memoirs, which are dated day by day in the form of poetry. 